\title{
Performance Evaluation of a Virtual Impactor with an Additional Pretreatment Structure for Particle Separation
}

\author{
Pan Wang ${ }^{1}$, Shouqi Yuan ${ }^{1 *}$, Ning Yang ${ }^{2 *}$, Aiying Wang ${ }^{3}$ \\ ${ }^{1}$ Research Center of Fluid Machinery Engineering and Technology, Jiangsu University, Zhenjiang \\ 212013, China \\ ${ }^{2}$ School of Electrical and Information Engineering, Jiangsu University, Zhenjiang 212013, China \\ ${ }^{3}$ China National Rice Research Institute, Hangzhou 310006, China
}

\section{ABSTRACT}

In this paper, a proposed method based on a microfluidic chip that can arrange the collected particles in radial positions according to their sizes before virtual impactor separation was introduced. The main objective of this paper was to design a novel micro-separator structure composed of two major parts: a virtual impactor, and a pretreatment that consists of a sheath flow and a backward-facing step flow configuration. In the separation process, the particles firstly were aggregated on the middle channel by the sheath flow, then ordered in radial position of backward-facing step flow, and finally separated in virtual impactor. To determine the effects of the pretreatment, both simulations and experiments were conducted. A parameter optimization was performed on the micro-separator where the cut-off size of the virtual impactor was $2.5 \mu \mathrm{m}$. The results demonstrated that when the flow ratio between inlet flow rate and the total flow rate was set to $50 \%$ and the expansion ratio $\left(E_{\mathrm{r}}\right)$ was 2 . The cut-off size of the micro-separator was reduced by $20.77 \%$ from $2.436 \mu \mathrm{m}$ to $1.93 \mu \mathrm{m}$, and the wall loss $(W L)$ was acceptable compared with the result only using $\mathrm{PM}_{2.5}$ virtual impactor. The effect of pretreatment on $\mathrm{PM}_{1}$ and $\mathrm{PM}_{5}$ virtual impactor also saw an improvement of collection efficiency even when the additional pretreatment had varying Reynold numbers and nozzle sizes. Accordingly, the stk $k_{50}$ value was reduced by $44.39 \%$ from 0.669 to 0.372 by adding pretreatment before the virtual impactor.

Received: June 19, 2020

Revised: October 22, 2020

Accepted: November 12, 2020

\section{${ }^{*}$ Corresponding Authors:}

Shouqi Yuan

shouqiy@ujs.edu.cn

Ning Yang

yangn@ujs.edu.cn

\section{Publisher:}

Taiwan Association for Aerosol Research

ISSN: $1680-8584$ print

ISSN: 2071-1409 online

(c) Copyright: The Author(s). This is an open access article distributed under the terms of the Creative Commons Attribution License (CC BY 4.0), which permits unrestricted use, distribution, and reproduction in any medium, provided the original author and source are cited.
Keywords: Microfluidics, Pretreatment, Particle arrangement, Virtual impactor, Collection efficiency

\section{INTRODUCTION}

Bioaerosols such as viruses, bacteria, and fungal spores pose a great threat to human lives and health, especially for patients with respiratory diseases (Blachere et al., 2009; Tsuda et al., 2013; Cui et al., 2015). The dispersed bioaerosols can also lead to an outbreak of epidemics in some areas due to the quick spread of fungi and viruses (Nasrabadi et al., 2017). Therefore, a highefficiency separation device is the foundation for detecting different sizes of particles.

Nowadays, owing to the simple operation and principle, virtual impactor has attracted much interest in aerosol science and widely used to classify particles according to their sizes (Marple et al., 1980; Zhang et al., 2016). To improve the collection efficiency of the impactor, various parameters of inertial impactor have been studied. Such as Reynolds number (Marple et al., 1974; Marple et al., 1975), the jet-to-plate distance (Marple et al., 1967; Grinshpun et al., 2005), the particle density (Huang et al., 2002), gravitational force (May et al., 1975; Huang et al., 2001) and etc. Besides, some novel structures have also been discovered to improve the collection efficiency curve. For example, Cheon et al. (2017) added an additional punched impaction plate between 
the nozzle outlet and the existing impaction plate to improve the collection efficiency and reduce the cut-off diameter by changing the particle trajectories. Lee et al. (2019) also proposed an inertial impactor with vertical annular nozzle to separate particles, as a result, it will increase the pressure slightly but increase collection efficiency significantly. However, there is also a significant problem called wall loss, it is caused by the trapped particles on the walls of the virtual impactor. To decrease the wall loss, a two-partitioned inlet which can supple both aerosol and clean air in particle separation process has been placed before the virtual impactor (Chen et al., 2016; Zahir et al., 2019; Lim et al., 2020), it can also adjust the flowrate ratios of aerosol to clean air to seek an optimal proportion to reduce the cut-off diameter efficiently.

Meanwhile, the combination of impactor and microfluidic chip has been used extensively to realize the airborne microorganism separation. Such as, Kang et al. (2012) proposed a microscale cascade impactor system for bioaerosol sampling and separation. Liu et al. (2016) presented a high-efficiency collection system based on microfluidic chip to measure the airborne mineral dust. Most importantly, impactor based on microfluidic chip can also overcome the limitations of bioaerosols real-time detection directly. For instance, Kang et al. (2014) integrated inertial impaction and mini-fluorescent microscopy to realize the airborne pathogenic microorganism's real-time detection successfully. Kim et al. (2009) integrated the electrode pair and virtual impactor based on microfluidic chip together to accelerate the electrical particle and improve the collection efficiency. Xu et al. (2013) observed the fine particle behavior successfully in microfluidic chip when it passed a triangular cylinder in a laminar airflow. Wang et al. (2020) replaced the impaction plate by a low-pressure collection chamber which eliminates the re-entrainment and bounce effects of particles, and finally confirmed a satisfied collection efficiency. However, most of the abovementioned studies does not include the improvement of the collection efficiency curve.

In this study, a novel separator structure based on microfluidic chip consists of two major parts: a virtual impactor, and a pretreatment that consists of a sheath flow and a backward-facing step flow configuration was proposed. To improve the collection efficiency curve and decrease wall loss rate, both simulations and experiments were performed to seek for optimum parameters. During the particle separation process, the distribution of the collected particles can realize an order pattern (the collected particles sequence according to their size in radial position) from a disordered pattern (all the particles were randomly distributed when they were collected) by the combination of a sheath flow and a backward-facing step flow. Then, the performance of the micro-separator was evaluated by detecting the monodispersed polystyrene latex (PSL) particles. It was expected that the new pretreatment structure can be applied to improve the performance of the existing virtual impactor, and enhance the measurement accuracy for the detection object assessment.

\section{MATERIALS AND METHOD}

\subsection{Micro-separator}

The detailed structure of the proposed micro-separator is schematically shown in Fig. 1(a), in which the micro-separator consists of pretreatment structure and virtual impactor, the pretreatment structure contains two parts including sheath flow area and backward-facing step flow area. In the working process, the aerosol sample with different particle sizes was firstly aggregated in the middle of the microchannel due to the sheath flow (Chen et al., 2016). Then, different aerosols were dispersed in a backward-facing step flow radial position according to their size. Therefore, the particle sequencing can be realized by the combination of sheath flow and backward-facing step flow. Finally, all particles were separated by the following virtual impactor. The results were validated by numerical analysis and compared to the experimental results. The geometric parameters of the proposed micro-separator are shown in Table 1 . In this section, the thickness of the microchannel was determined to be $160 \mu \mathrm{m}$ to prevent a strong Dean Vortex effect because the maximum sample flow rate is $96.5 \mathrm{~mL} \mathrm{~min}{ }^{-1}$. The parameters of the backwardfacing step flow were obtained by numerical simulation. The jet-to-plate distance was determined by the width of the injection nozzle $\left(W_{4}\right)$, and the $S / W_{4}$ ratio is within 1.2 to 1.8 (Loo et al., 1988). In this study, the width of the injection nozzle was set to $290 \mu \mathrm{m}$, so the jet-to-plate distance was $435 \mu \mathrm{m}$. The photograph of the micro-separator is shown in Fig. 1(b). In addition, to analyze the 
(a)

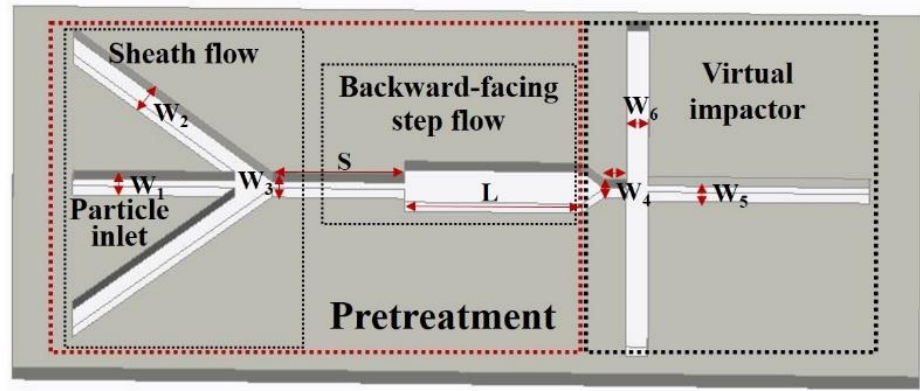

(b)

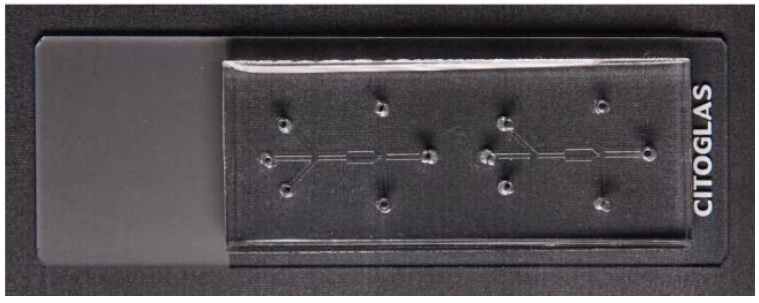

Fig. 1. (a) Schematic of the proposed micro-separator. The micro-separator consists of two parts including pretreatment and virtual impactor, and the pretreatment was separated into sheath flow and back-forward facing step flow. (b) The photograph of the micro-separator.

Table 1. The geometric parameters of the proposed micro-separator.

\begin{tabular}{lc}
\hline Description & Value $(\mu \mathrm{m})$ \\
\hline The width of inlet $\left(W_{1} / W_{2}\right)$ & 500 \\
The thickness of the microchannel & 160 \\
The width of the channel between the sheath flow outlet and the Backward-facing step flow inlet $\left(W_{3}\right)$ & 500 \\
The distance between the sheath flow outlet and the Backward-facing step flow inlet $(S)$ & 5000 \\
The width/length of the injection nozzle $\left(W_{4}\right)$ & 290 \\
The length of the backward-facing step flow $(\mathrm{L})$ & 5000 \\
The width of the minor/major flow $\left(W_{5} / W_{6}\right)$ & 435 \\
\hline
\end{tabular}

performances of the collection efficiency and the $W L$ of the proposed micro-separator, additional enrichment microfluidic chips, as shown in Fig. S1, were placed after the outlets of the microseparator to collect the separated particles. In this process, the retention of the particles in the micro-separator was recorded as $W L$, and the particles collected in the enrichment microfluidic chip was used to calculate the collection efficiency of the micro-separator. The enrichment microfluidic chip comprised an inlet, a collection area, and an outlet.

\subsection{Pretreatment}

In the first part of pretreatment, the sheath flow was set symmetrically to squeeze the sample flow into the middle of the convergence channel, as shown in Fig. 2(a). The sheath flow also has a great impact on reducing the $W L$ and the influences caused by the random distribution of the initial position due to the particle aggregation effect (Chen et al., 2016). In the second part of pretreatment, the backward-facing step flow was designed after sheath flow. The working mechanism of the backward-facing step flow can be explained as follows: the trajectories of particles past the backward-facing step flow vary from the streamline due to particle inertia which is mainly determined by the particle size. Particles with different sizes that past the backwardfacing step flow were shown in Fig. 2(b), it was observed that, due to the strong inertia force and radial drag force, particle trajectories will diverge in terms of their sizes in radial position, therefore, small particles have greater tendency to follow the airflow, while the trajectories of larger ones will not change due to the sufficient particle inertia (Klemz et al., 2017). As a result, particles collected by micro-separator will be separated in radial position. Here, particles with different sizes experienced different focusing patterns and exhibited different trajectories. 

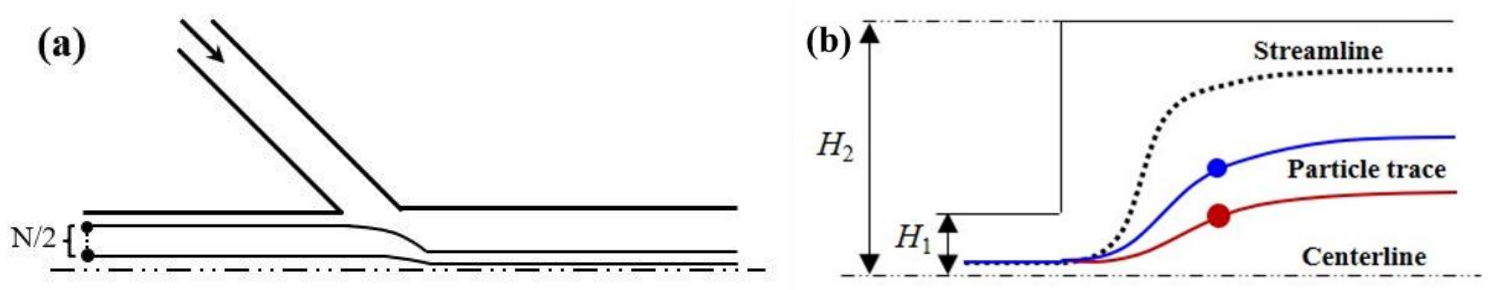

Fig. 2. (a) The trajectories of particles in the sheath flow. In this figure, $\mathrm{N}$ represents the number of particles that flow into the particle inlet. (b) The trajectories of particles in the backward-facing step flow.

Additionally, the separation efficiency was influenced by the expansion ratio $\left(E_{r}\right)$, which was defined as Eq. (1),

$E_{r}=H_{2} / H_{1}$

In this study, $H_{1}$ is the half-width of the particle inlet, $H_{2}$ is the distance between centerline and boundary of the backward-facing step flow.

\subsection{Virtual Impactor}

In this section, the virtual impactor is an aerodynamic device that classifies particles in terms of their size. The working principle of the virtual impactor is simple, when particles flow into the virtual impactor, and the majority particles larger than the cut-off size are collected in the minor channel, the majority particles smaller than the cut-off size are passed through (The cut-off size is the aerodynamic diameter with a collection efficiency of 50\%) (Kim et al., 2009). In the virtual impactor design, their aerodynamic diameter was defined as (Lee et al., 2019):

$d_{a}=d_{p} \sqrt{\frac{\rho_{p} C_{c}\left(d_{p}\right)}{\chi \rho_{a} C_{c}\left(d_{a}\right)}}$

where $d_{\mathrm{p}}$ is particle size, $\rho_{\mathrm{p}}$ is the particle density, $\chi$ is the dynamic shape factor, $\rho_{\mathrm{a}}$ is the standard particle density (i.e., $\left.1000 \mathrm{~kg} \mathrm{~m}^{-3}\right)$, and $C_{\mathrm{c}}\left(d_{\mathrm{p}}\right)$ is the Cunningham slip correction factor (Kang et al., 2014), which can be expressed by Eq (3):

$C_{c}=1+0.5 K n_{p}\left[2.34+1.05 \exp \left(-0.195 K n_{p}\right)\right]$

where $K n_{p}$ is particle Knudsen number, which is defined as $2 \lambda / d_{p}$, where $\lambda$ is the mean free path of the air molecule. The Stokes number (Kang et al., 2014) was defined as the following Eq. (4).

$s t k=\frac{\rho_{P} d_{p}^{2} C_{c} V}{9 \mu W}$

where $V$ is the air velocity at the inlet, $\mu$ is the air viscosity, $W$ is the hydraulic diameter of the nozzle. Then, the cut-off size $\left(d_{50}\right)$ can be obtained by Eq. (5).

$d_{50}=\sqrt{\frac{9 \mu W s t k_{50}}{\rho_{P} C_{C} V}}$

where $s t k_{50}$ represents the Stokes number that corresponds to $50 \%$ particle collection efficiency.

\subsection{Numerical Analysis}

The effects of pretreatment on particle separation were evaluated by using computational fluid dynamics (CFD) simulation. In this study, COMSOL Multiphysics was utilized for fluid flow simulation. 
The simulation conditions were set to a steady, two-dimensional simulation model under laminar and incompressible flow, which can quickly provide insights into the physical phenomena of the designed micro-separator. The particle density was set at $1050 \mathrm{~kg} \mathrm{~m}^{-3}$. In this design, the cut-off sizes of $\mathrm{PM}_{1}, \mathrm{PM}_{2.5}$, and $\mathrm{PM}_{5}$ were obtained with the same virtual impactor by changing the flow rate. According to the simulation results, the flow rates of the major and minor flow in $\mathrm{PM}_{1}, \mathrm{PM}_{2.5}$, and $\mathrm{PM}_{5}$ were set as: $45 \mathrm{~mL} \mathrm{~min}{ }^{-1}$ and $6.5 \mathrm{~mL} \mathrm{~min}^{-1}, 9 \mathrm{~mL} \mathrm{~min}^{-1}$, and $1 \mathrm{~mL} \mathrm{~min}^{-1}, 1.8 \mathrm{~mL} \mathrm{~min}^{-1}$ and $0.3 \mathrm{~mL} \mathrm{~min}^{-1}$, respectively, according, the flow rate ratios between the major flow and minor flow are about 7, 9 and 6 , respectively. Then, the micro-separator was also simulated by changing these flow rates to verify the pretreatment effects, and the flow rate of the particle inlet was set to half of the total flow rate. The boundary condition of sheath flow inlet was set as pressureinlet, and the other boundary conditions were set as no-slip wall. Smaller grid sizes can provide more accurate simulation results, but the greater the number of the grids, the longer the simulation time takes. Therefore, it is important to select suitable grids number to simulate, in this study, about 20000 grids were used. During the simulation, the particle samples with sizes of $0.2,0.5,0.7,0.8,0.9,1,1.1,1.5,1.8,2,2.5,3,3.5,4,4.5,5,5.5,6.0,7.0,8.0,9.0$ and $10 \mu \mathrm{m}$ were chosen, and 500 particles with uniform size and density were arranged at regular intervals from the center of particle inlet to the edge. Then, calculate the flow field, and the discrete phase model (DPM) with the Lagrangian approach was applied to simulate particle trajectories. The parametric studies were performed to optimize the pretreatment structure by varying the ratio of the inlet flow rate and total flow rate and changing the $E_{\mathrm{r}}$ of the backward-facing step flow. Finally, the particles collected in the major and minor flow were counted respectively, and the collection efficiency of a given particle size is calculated using the following equation:

$E(\%)=\frac{N_{\text {Minor }}}{N_{\text {Major }}+N_{\text {Minor }}} \times 100 \%$

where $E(\%), N_{\text {Major, and }} N_{\text {Minor }}$ are the collection efficiency (\%), the numbers of the given particle size collected in the major flow, and minor flow, respectively. Then, the $W L$ of a given particle size can be calculated from Eq. (7):

$W L=\left(1-\frac{N_{\text {Major }}+N_{\text {Minor }}}{N_{\text {Total }}}\right) \times 100 \%$

where $N_{\text {Total }}$ is the total number of the given particle size in the micro-separator and the enrichment microfluidic chip.

\subsection{Experimental}

Fig. 3 shows a schematic diagram of the experimental setup for the performance evaluation of the virtual impactor and the corresponding micro-separator. PSL spheres with diameters of 0.2 , $0.5,0.8,1.0,1.5,2.0,2.5,3.0$, and $4.0 \mu \mathrm{m}$ were used, the density of PSL particle is $1050 \mathrm{~kg} \mathrm{~m}^{-3}$. Firstly, the air was injected by the compressor through the HEPA filter into the aerosol generator (24 Jet Collison, BGI Collison) under the 3 atm for atomizing bioaerosols. Subsequently, the PSL particles flow through a diffusion dryer to remove the moisture contained in the particles, and a ${ }^{210}$ Po neutralizer was placed after the diffusion dryer to remove the electrical charge of the particles. Thirdly, the sample flow into the microfluidic chip from the particle inlet at a fixed flow rate which was controlled by rotameter (1-10 $\mathrm{mL} \mathrm{min}^{-1}, 6-60 \mathrm{~mL} \mathrm{~min}^{-1}$, measurement uncertainty is less than $0.66 \%$ ), the inlets of sheath flow were connected with the HEPA filter, the excess flow was vented to the atmosphere. Particles that passed through the micro-separator were collected by enrichment microfluidic chip. Moreover, the whole flow in the microfluidic chip was controlled by three vacuum pumps which were placed after enrichment microfluidic chip. Finally, the polydimethylsiloxane (PDMS) of micro-separator and enrichment microfluidic chip were removed in the laboratory, only the microfluidic chip substrates were left and put in the scanning electron microscope (SEM, Hitachi, S-3400N) after the gold sputtering treated. Then, the images of the whole microfluidic channel were taken by SEM, and the size distribution of PSL particles was calculated based on Image Processing and Recognition. 


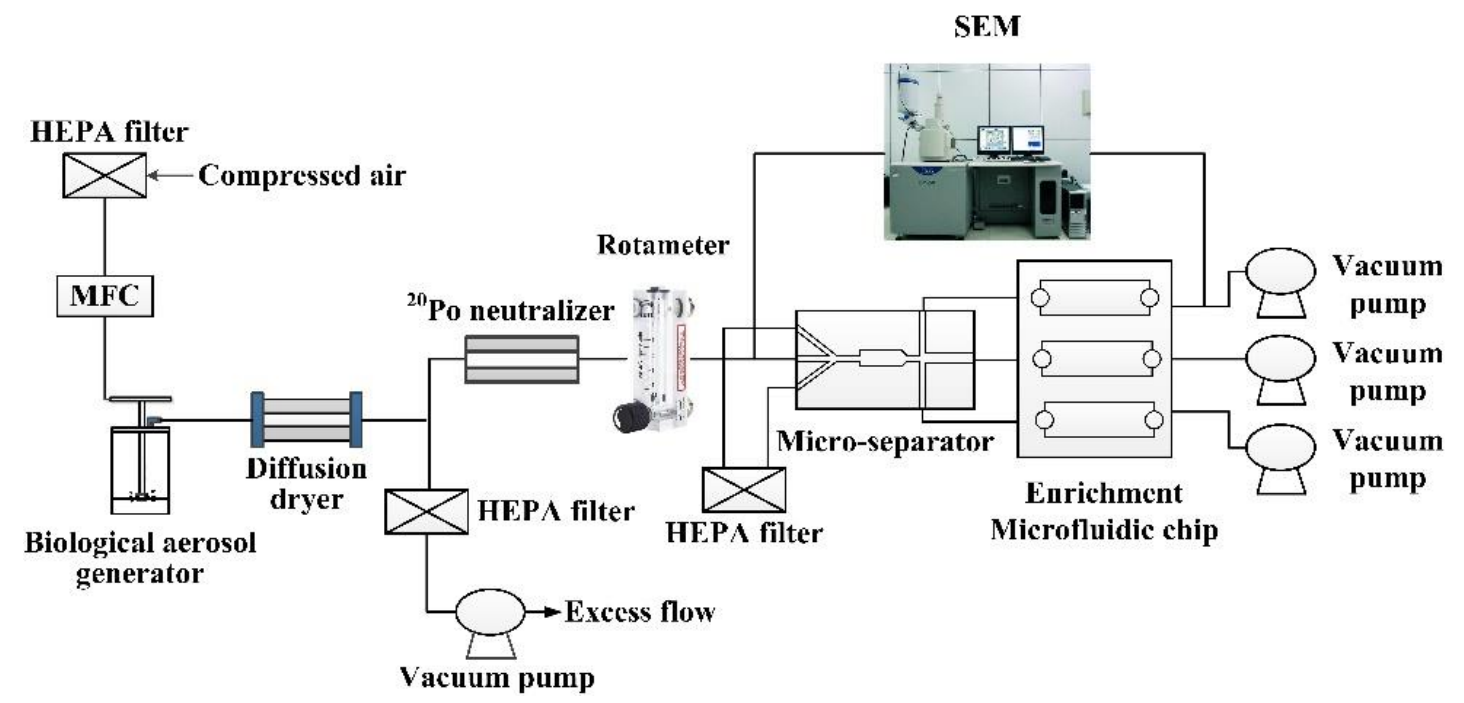

Fig. 3. Schematic of the experimental setup.

\section{RESULTS AND DISCUSSION}

\subsection{The Optimal Parameters of Sheath Flow and Backward-facing Step Flow}

In this section, the flow ratio was defined as the flow rate of particle inlet to the total flow rate of the microfluidic chip, and $E_{\mathrm{r}}$ were optimized based on the micro-separator, in which the cut-off size of the virtual impactor is $2.5 \mu \mathrm{m}$.

In the sheath flow area, the sheath flow angle was $45^{\circ}$ (Chen et al., 2016). Particle trajectories were aggregated by the squeeze phenomenon caused by the sheath flow at the final state. As shown in Fig. 4(a), with increasing the flow ratio, decreased small particle collection efficiency was observed in the minor flow channel. The particle aggregation is beneficial to reduce the cutoff size, however, it has an obvious negative influence that the small particles (smaller than the cut-off size) collection efficiency is high when the flow ratio is small, which could significantly affect the target detection precision. Therefore, taking the small particle collection efficiency (lower than 16\%) into consideration, the flow ratio equals 50\%. Besides, the Major and Minor ratio which was defined as the flow rate of the major flow rate to the minor flow rate has been also analyzed. At a fixed total flow rate $\left(93.5 \mathrm{~mL} \mathrm{~min}^{-1}\right)$, there are four Major and Minor ratios that have been studied, as shown in Fig. 4(b), the cut-off size reduced when the ratio decreases from 10 to 4 , however, the collection efficiency of relatively small particles increases with the decrease of the ratio. Then, as presented in Fig. 4(c), the variation in cut-off sizes can be obtained by changing $\mathrm{H}_{2}$ based on the proposed micro-separator. The result shows that the cut-off size decreases with the $H_{2}$ increasing firstly, and it changes slightly when $H_{2}$ is larger than $0.5 \mathrm{~mm}$. Therefore, $H_{2}$ was set to $0.5 \mathrm{~mm}$, accordingly, the $E_{\mathrm{r}}$ was 2 .

\subsection{The Collection Efficiency and WL of the Micro-separator}

In this section, the traditional $\mathrm{PM}_{1}$ and $\mathrm{PM}_{5}$ virtual impactor and the corresponding microseparators were also generated based on different flow rate settings. The collection efficiency curves of the traditional virtual impactor and the corresponding micro-separator obtained from the experiments were shown in Fig. 5. According to the $\mathrm{PM}_{1}$ impactor results in Fig. 5(a), the cutoff size was $0.989 \mu \mathrm{m}$ without the combination of sheath flow and backward-facing step flow, and it was reduced to $0.759 \mu \mathrm{m}$ by adding an extra pretreatment. Likewise, as shown in Fig. 5(b), the cut-off size of the $\mathrm{PM}_{2.5}$ impactor was reduced from $2.436 \mu \mathrm{m}$ to $1.93 \mu \mathrm{m}$ through by applying the pretreatment. Since PSL particles larger than $4 \mu \mathrm{m}$ were difficult to be atomized, therefore, $\mathrm{PM}_{5}$ impactor with or without pretreatment was compared by using the simulation method, as shown in Fig. 5(c), the cut-off size was reduced from $4.807 \mu \mathrm{m}$ to $2.981 \mu \mathrm{m}$. Furthermore, the sharpness $(\sigma)$ of the experimental curve was evaluated by the following Eq. (8) (Hinds et al., 1999). 
(a)

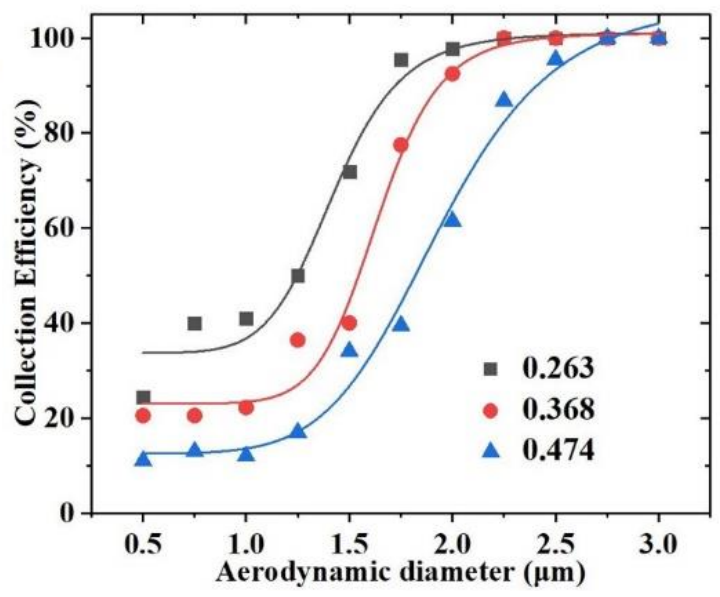

(c)

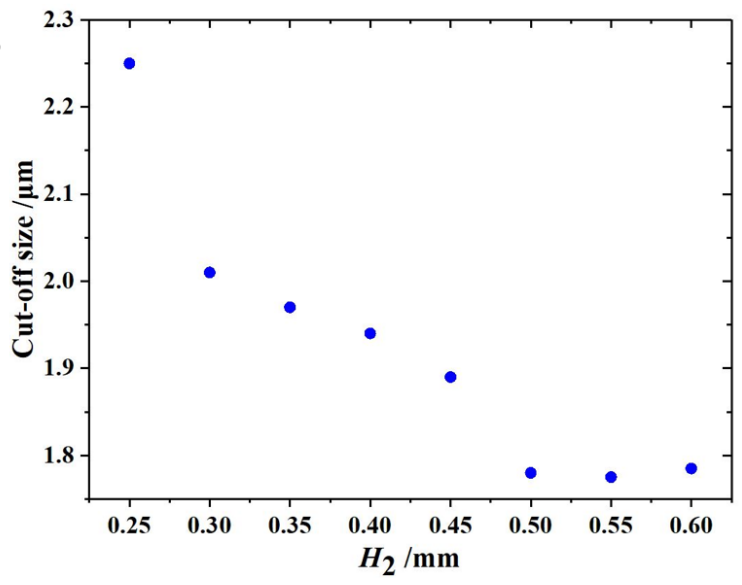

(b)

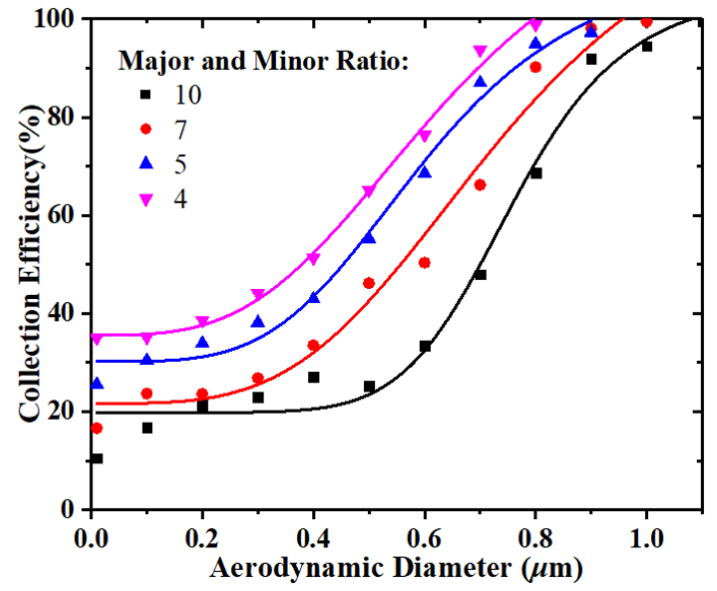

Fig. 4. Variation in cut-off size of the proposed micro-separator obtained by numerical results: (a) The effect of the flow ratio, (b) The influence of the Major and Minor Ratio, (c) The optimization of the backward-facing step flow.

$$
\sigma=\sqrt{\frac{d_{84}}{d_{16}}}
$$

where $\sigma$ is the geometric standard deviation. $d_{84}$ and $d_{16}$ are the particle size with a collection efficiency of $84 \%$ and $16 \%$, respectively. The GSDs of micro-separator in three different conditions were $1.24,1.189,1.263$. The sharpness of the collection efficiency curve is higher than the curve without the pretreatment, the reason is that the particle stream will be squeezed in the middle of the microchannel when the clean air was injected from the sheath flow, then the particles with relatively small size will tend to close the edge of microchannel in backward-facing step flow, therefore, the particles with different sizes will be ordered firstly in the pretreatment structure before the virtual impactor separation, the collection efficiency will be improved compared to the disordered particles. Besides, the $W L$ of the proposed micro-separator with different flow ratio were calculated by using Eq. (7). As shown in Fig. $5(\mathrm{~d})$, the $W L$ is less than $20 \%$, which is acceptable (Marple et al., 1991; Hyun et al., 2018). Thus, the additional pretreatment was confirmed to improve the collection efficiency curve.

To investigate the cut-off size reduction mechanism of the virtual impactor with an additional pretreatment, the flow streamlines and particle trajectories in the impactor were simulated and observed in Fig. 6. In the case of virtual impactor without the pretreatment, as shown in Fig. 6(a), the flow streamlines were evenly distributed. Meanwhile, as shown in Fig. 6(b), all the particles were randomly distributed, a part of particles with enough inertia force flow into the minor flow and the others can change direction by $90^{\circ}$ after being accelerated through the nozzle. In the case of the micro-separator, the flow streamlines were focused on the centerline firstly, then it will disperse at both sides of the channel, as shown in Fig. 6(c). The Stokes number and the inertia 
(a)

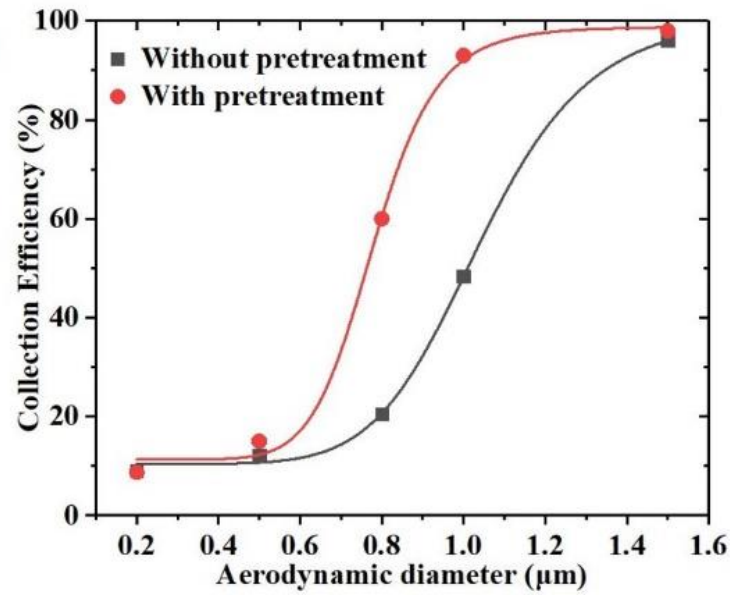

(c)

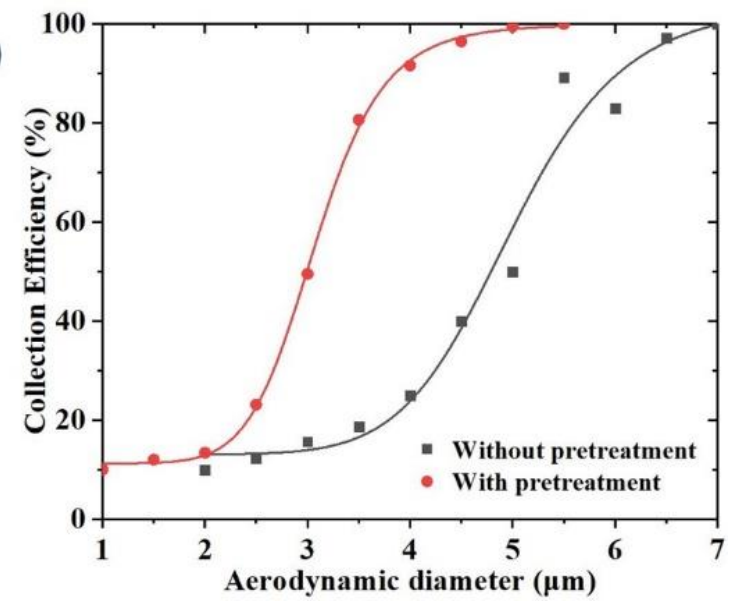

(b)

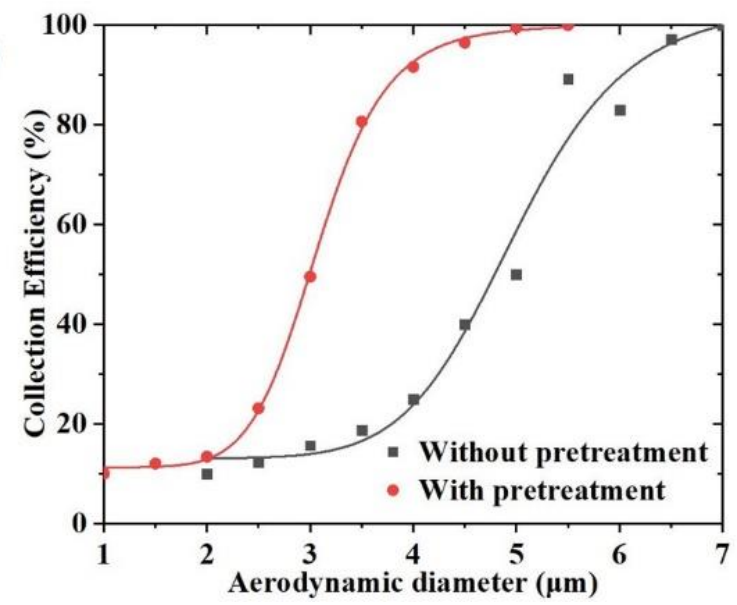

(d)

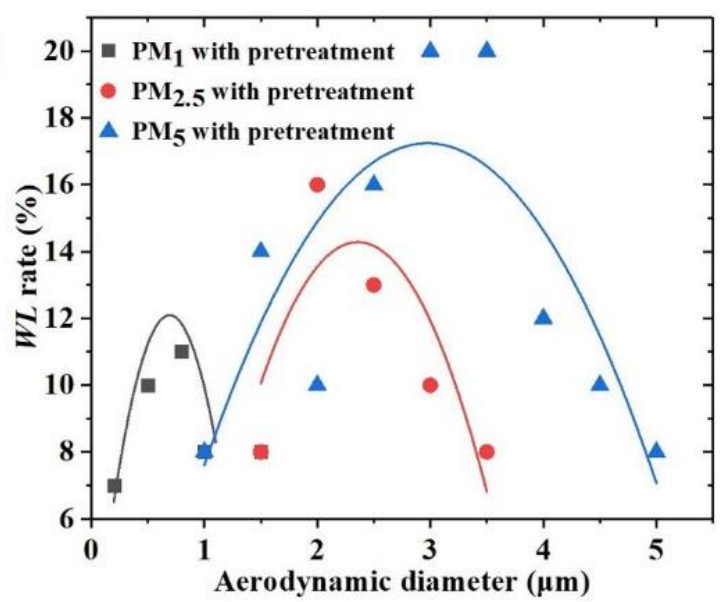

Fig. 5. The collection efficiency curves of the virtual impactor and the proposed micro-separator. (a) The collection efficiency curves of the traditional $\mathrm{PM}_{1}$ virtual impactor and the corresponding micro-separator obtained from the experimental data; (b) The collection efficiency curves of the traditional $\mathrm{PM}_{2.5}$ virtual impactor and the corresponding micro-separator obtained from the experimental data; (c) The simulations of the traditional $\mathrm{PM}_{5}$ virtual impactor and the corresponding micro-separator obtained from the numerical results; (d) The $W L$ rate of the proposed micro-separator. In this figure, the $W L$ rate of the $\mathrm{PM}_{1}$ and $\mathrm{PM}_{2.5}$ with pretreatment were experimental results, the $\mathrm{PM}_{5}$ with pretreatment were numerical results.

force are accordingly different due to different cut-off size, the particles will diffuse according to the resultant force of radial drag force and inertia force, so the particle disperses system with the fixed trend will be formed. Due to these flows, the particles can be ordered in the middle area before the virtual impactor, as shown in Fig. 6(d), the collection efficiency was enhanced effectively by coupling the sheath flow and backward-facing step flow.

In summary, the sheath flow and the backward-facing step flow complement each other. The sheath flow has significant effects on the reduction of cut-off size by aggregating the particles in the middle of the micro-channel. Meanwhile, the setting of backward-facing step flow provides a radial drag force which is beneficial for solving the problem that small particles with high collection efficiency caused by sheath flow. By applying this pretreatment, the particle sequence was ordered according to their sizes, the cut-off size was reduced and a satisfying collection efficiency curve was also obtained.

Fig. 7 shows the simulation results of the different virtual impactor with or without the pretreatment. The Stokes number is indicated in the x-axis of the graph. The width of the nozzle, aerosol flow rate, and the corresponding nozzle Reynolds number of various virtual impactor models used in the numerical parametric study are presented in Table 2. Because the collection efficiency relates to Stokes number, the fitting curve of the virtual impactor with or without the additional pretreatment can be obtained from each case. From the fitting curve comparison, Stk $k_{50}$ is 0.669 when only using the traditional virtual impactor; $S t k_{50}$ is 0.372 when the additional 

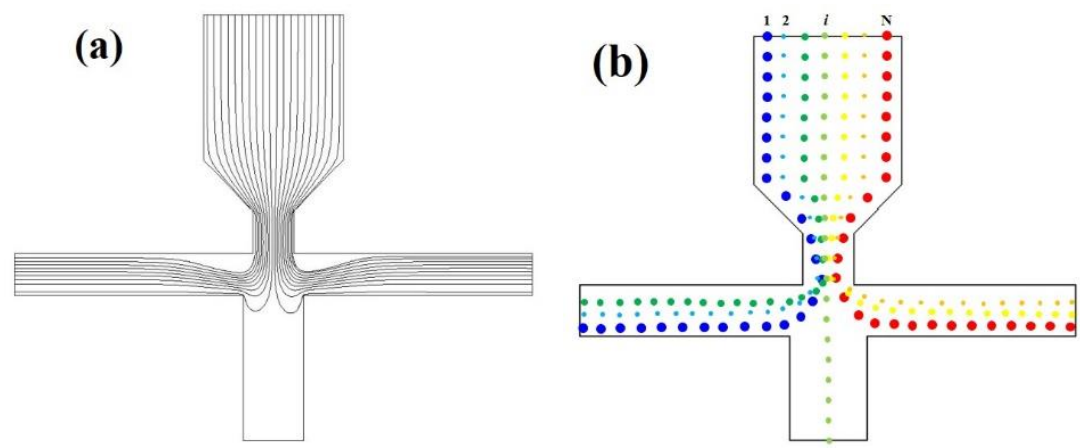

(c)

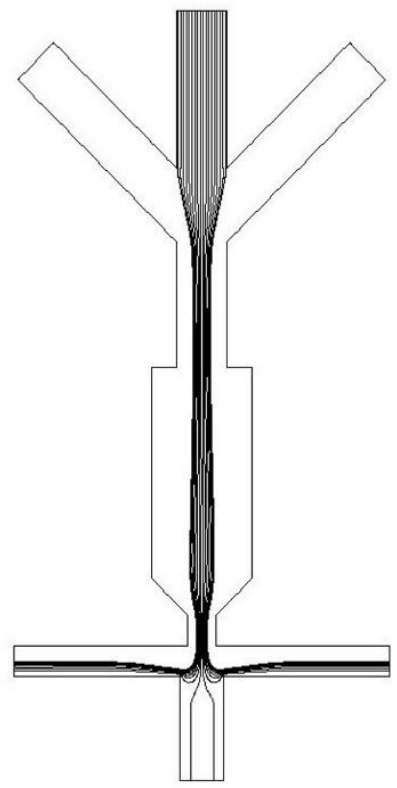

(d)

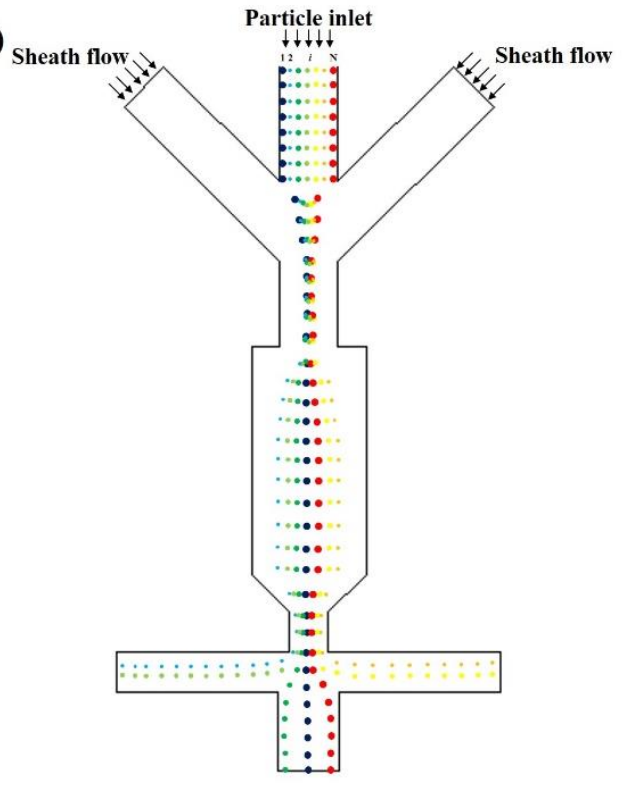

Fig. 6. Comparison between virtual impactors with and without the additional coupling structure: (a) and (b) are streamline and particle trajectory of the traditional virtual impactor, respectively. (c) and (d) are streamline and particle trajectory of micro-separator, respectively.

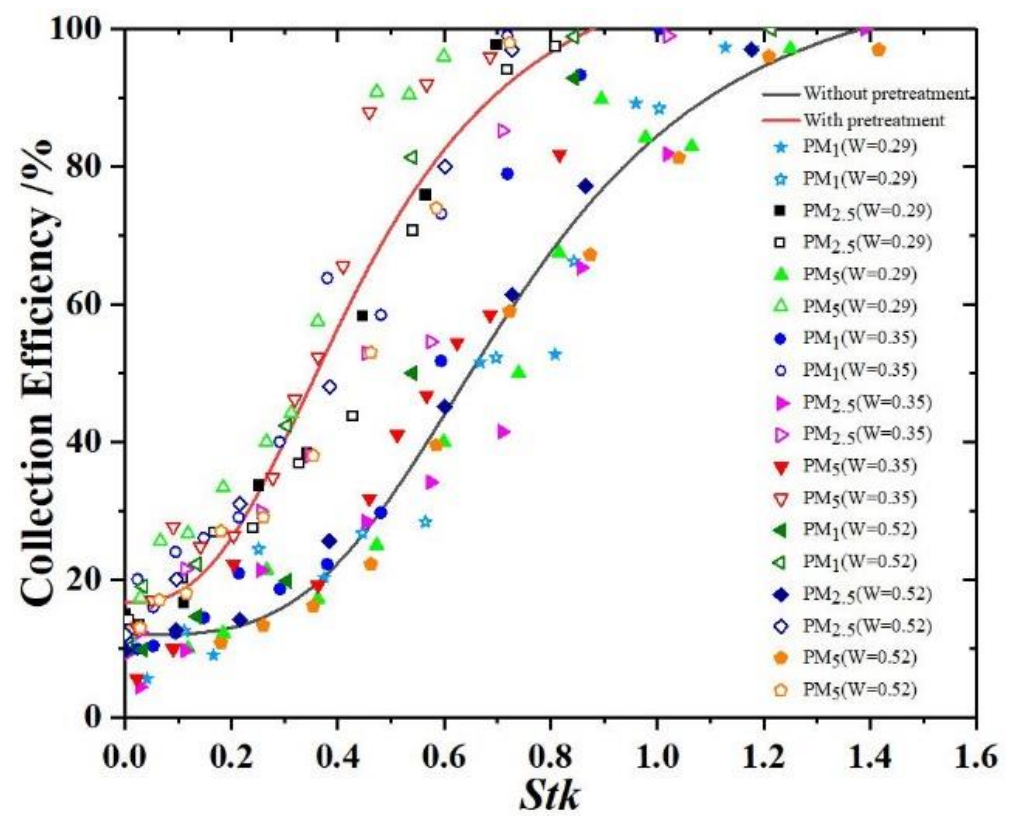

Fig. 7. The comparison of the collection efficiency curves of the virtual impactor and the proposed micro-separator obtained from the numerical data. 
Table 2. Nozzle sizes and aerosol flow rates assumed for the numerical parametric study.

\begin{tabular}{|c|c|c|c|c|}
\hline The width of nozzle (mm) & Cut-off size $(\mu \mathrm{m})$ & Inlet flow rate $\left(\mathrm{mL} \mathrm{min}^{-1}\right)$ & Total flow $\left(\mathrm{mL} \mathrm{min}^{-1}\right)$ & Nozzle Reynolds Number \\
\hline \multirow[t]{3}{*}{0.29} & 1 & 45 & 88 & 465.17 \\
\hline & 2.5 & 7.5 & 14.7 & 77.7 \\
\hline & 5 & 2 & 3.9 & 21.19 \\
\hline \multirow[t]{3}{*}{0.35} & 1 & 47.5 & 99.5 & 465.35 \\
\hline & 2.5 & 11 & 21.75 & 101.68 \\
\hline & 5 & 2.95 & 5.5 & 25.77 \\
\hline \multirow[t]{3}{*}{0.52} & 1 & 115.75 & 227.5 & 797.56 \\
\hline & 2.5 & 20.5 & 41 & 143.69 \\
\hline & 5 & 6.5 & 12.25 & 42.88 \\
\hline
\end{tabular}

pretreatment was added, showing a decrease of $44.39 \%$. According to Eq. (8), the sharpness of the collection efficiency curve was estimated to be 1.384 without the additional pretreatment, while the sharpness was 1.577 with the additional pretreatment, showing an increase of $13.6 \%$.

\section{CONCLUSION}

In this study, a novel method enabling particles to be arranged firstly according to the sizes before the virtual impactor separation was proposed to improve the collection efficiency curve of the traditional virtual impactor. Accordingly, a novel micro-separator was designed which consists of sheath flow, backward-facing flow, and traditional virtual impactor. As a result, the flow ratio and $\mathrm{H}_{2}$ were optimized to $50 \%$ and $0.5 \mathrm{~mm}$, respectively. The cut-off size of the proposed micro-separator was smaller than the traditional virtual impactor, and it reduces from 0.989 to $0.759 \mu \mathrm{m}$ for $\mathrm{PM}_{1}$ virtual impactor, 2.436 to $1.93 \mu \mathrm{m}$ for $\mathrm{PM}_{2.5}$ virtual impactor, and 4.807 to $2.981 \mu \mathrm{m}$ for $\mathrm{PM}_{5}$ virtual impactor. Again, the fitting curves could be observed from the collection efficiency data, resulting in $S t k_{50}=0.669$ in traditional virtual impactor and $S t k_{50}=0.372$ with the additional pretreatment, a decrease of approximately $44.39 \%$ was achieved. In conclusion, the development of the proposed micro-separator can be applied to increase the particle collection efficiency and enhance the measurement accuracy for target assessment.

\section{ACKNOWLEDGMENTS}

This work was supported by the Jiangsu Agricultural Science and Technology Innovation Fund (grant numbers CX (18)3043), National Key Research and Development Project (grant numbers 2019YFC1606600), Outstanding Youth Science Foundation of Jiangsu Province (grant numbers BK20180099), Open project of the State Key Laboratory of Rice Biology (grant number 20200302), Project of Agricultural Equipment Department of Jiangsu University (grant number NZXB20200205).

\section{CONFLICTS OF INTEREST}

The authors declare no conflict of interest.

\section{SUPPLEMENTARY MATERIAL}

Supplementary data associated with this article can be found in the online version at https://doi.org/10.4209/aaqr.2020.05.0269

\section{REFERENCES}

Blachere, F.M., Lindsley, W.G., Pearce, T.A., Anderson, S.E., Fisher, M., Khakoo, R., Meade, B.J., Lander, O., Davis, S., Thewlis, R.E., Celik, I., Chen, B.T., Beezhold, D.H. (2009). Measurement of 
airborne influenza virus in a hospital emergency department. Clin. Infect. Dis. 48, 438-440. https://doi.org/10.1086/596478

Chen, H.Y., Huang, H.L. (2016). Numerical and experimental study of virtual impactor design and aerosol separation. J. Aerosol Sci. 94, 43-55. https://doi.org/10.1016/j.jaerosci.2015.12.001

Cheon, T.W., Lee, J.Y., Bae, J.Y., Yook, S.J. (2017). Enhancement of collection efficiency of an inertial impactor using an additional punched impaction plate. Aerosol Air Qual. Res. 17, 2349-2357. https://doi.org/10.4209/aaqr.2017.01.0018

Cui, S., He, Z.Z., Zhu, Z.W., Sun, Z., Xu, Y.T., Wang, J.L., Bao, Y.Y., Ji, D.Y., Liu, S., Liu, J.T., Zhang, J.H., Wang, Q. (2015). Microfluidic analysis of $\mathrm{PM}_{2.5}$-induced epithelial-mesenchymal transition in human bronchial epithelial 16HBE cells. Microfluid. Nanofluid. 19, 263-272. https://doi.org/10. 1007/s10404-014-1499-3

Grinshpun, S.A., Mainelis, G., Trunov, M., Górny, R.L., Sivasubramani, S.K., Adhikari, A., Reponen, T. (2005). Collection of airborne spores by circular single-stage impactors with small jet-to-plate distance. J. Aerosol Sci. 36, 575-591. https://doi.org/10.1016/j.jaerosci.2004.06.078

Hinds, W.C. (1999). Aerosol technology: Peoperties, behavior, and measurement of airborne particle, 2nd edn, Wiley, New York.

Huang, C.H., Tsai, C.J. (2001). Effect of gravity on particle collection efficiency of inertial impactors. J. Aerosol Sci. 32, 375-387. https://doi.org/10.1016/S0021-8502(00)00086-0

Huang, C.H., Tsai, C.J. (2002). Influence of impaction plate diameter and particle density on the collection efficiency of round-nozzle inertial impactors. Aerosol Sci. Technol. 36, 714-720. https://doi.org/10.1080/02786820290038410

Hyun, J., Han, J., Lee, S.G., Hwang, J. (2018). Design and performance evaluation of a PN1 sensor for real-time measurement of indoor aerosol size distribution. Aerosol Air Qual. Res. 18, 285300. https://doi.org/10.4209/aaqr.2017.08.0263

Kang, J.S., Lee, K.S., Lee, K.H., Kim, S.S., Bae, G.N., Jung, J.H. (2014). Real-time detection of an airborne microorganism using inertial impaction and mini-fluorescent microscopy. Lab Chip 14 244-251. https://doi.org/10.1039/c3lc50805f

Kang, J.S., Lee, K.S., Lee, K.H., Sung, H.J., Kim, S.S. (2012). Characterization of a microscale cascade impactor. Aerosol Sci. Technol. 46, 966-972. https://doi.org/10.1080/02786826.2012.685115

Kim, Y.H., Park, D., Hwang, J., Kim, Y.J. (2009). A hybrid chip based on aerodynamics and electrostatics for the size-dependent classification of ultrafine and nano particles. Lab Chip 9, 2722-2728. https://doi.org/10.1039/b902211b

Klemz, A.C., Fontana, E., de Souza, A.A.U., de Souza, S.M.A.G.U. (2017). Analysis of heat and mass transfer in diffusion flame reactors coupled with aerodynamic lenses. Chem. Eng. Res. Des. 118 215-225. https://doi.org/10.1016/j.cherd.2016.10. 045

Lee, H., Sung, G., Kim, T., Shin, W.G. (2019). Novel in-line aerosol impactor utilizing upward inlet flow. J. Aerosol Sci. 129, 87-97. https://doi.org/10.1016/j.jaerosci.2018.12.005

Lim, J.H., Park, D., Yook, S.J. (2020). Development of a multi-slit virtual impactor as a high-volume bio-aerosol sample. Sep. Purif. Technol. 250, 117275. https://doi.org/10.1016/j.seppur.2020.1 17275

Liu, F., Han, Yu., Du, L., Huang, P.F., Zhe, J. (2016). Airborne mineral dust measurement using an integrated microfluidic device. Microfluid. Nanofluid. 20, 27. https://doi.org/10.1007/s10404015-1672-3

Loo, B.W., Cork, C.P. (1988). Development of high efficiency virtual impactors. Aerosol Sci. Technol. 9, 167-176. https://doi.org/10.1080/02786828808959205

Marple, V.A., Willeke, K. (1967). Impactor design. Atmos. Environ. 10, 891-896. https://do i.org/10.1016/0004-6981(76)90144-X

Marple, V.A., Liu, B.Y.H. (1974). Characteristics of laminar jet impactors. Environ. Sci. Technol. 8 648-654. https://doi.org/10.1021/es60092a003

Marple, V.A., Liu, B.Y.H. (1975). On the fluid flow and aerosol impaction in inertial impactors. J. Colloid Interface Sci. 53, 31-34. https://doi.org/10.1016/0021-9797(75)90031-4

Marple, V.A., Chien, C.M. (1980). Virtual impactors: A theoretical study. Environ. Sci. Technol. 14, 976-985. https://doi.org/10.1021/es60168a019

Marple, V.A. Rubow, K.L., Behm, S.M. (1991). A microorifice uniform deposit impactor (MOUDI): Description, calibration, and use. Aerosol Sci Technol. 14, 434-446. https://doi.org/10.1080/0 2786829108959504 
May, K.R. (1975). Aerosol impaction jets. J. Aerosol Sci. 6, 603-411. https://doi.org/10.1 016/0021-8502(75)90056-7

Nasrabadi, A.M., Park, J.W., Kim, H.S., Han, J.S., Hyun, J. Yong, D., Hwang, J. (2017). Assessment of indoor bioaerosols using a lab-made virtual impactor. Aerosol Sci. Technol. 51, 159-167. https://doi.org/0.1080/02786826.2016.1246707

Tsuda, A., Henry, F.S., Butler, J.P. (2013). Particle transport and deposition: Basic physics of particle kinetics. Compr. Physiol. 3, 1437-1471. https://doi.org/10.1002/cphy.c100085

Wang, P., Yuan, S.Q., Yang, N., Wang, A.Y., Fordjour, A., Chen, S.B. (2020). The collection method for crop fungal spores based on an efficient microfluidic device. Aerosol Air Qual. Res. 20, 7279. https://doi.org/10.4209/aaqr.2019.08.0424

Xu, J.Y., Zhang, J., Wang, H., Mi, J.C. (2013). Fine particle behavior in the air flow past a triangular cylinder. Aerosol Sci. Technol. 47, 875-884. https://doi.org/10.1080/02786826.2013.798612

Zahir, M.Z., Heo, J.E., Yook, S.J. (2019). Influence of clean air and inlet configuration on the performance of slit nozzle virtual impactor. Adv. Powder Technol. 30, 3224-3230. https://doi.org/10.1016/j.apt.2019.09.031

Zhang, C., Zhu, R., Yang, W. (2016). A micro aerosol sensor for the measurement of airborne ultrafine particles. Sensors 16, 399-407. https://doi.org/10.3390/s16030399 